International Journal of Medical Anesthesiology 2019; 2(2): 89-90

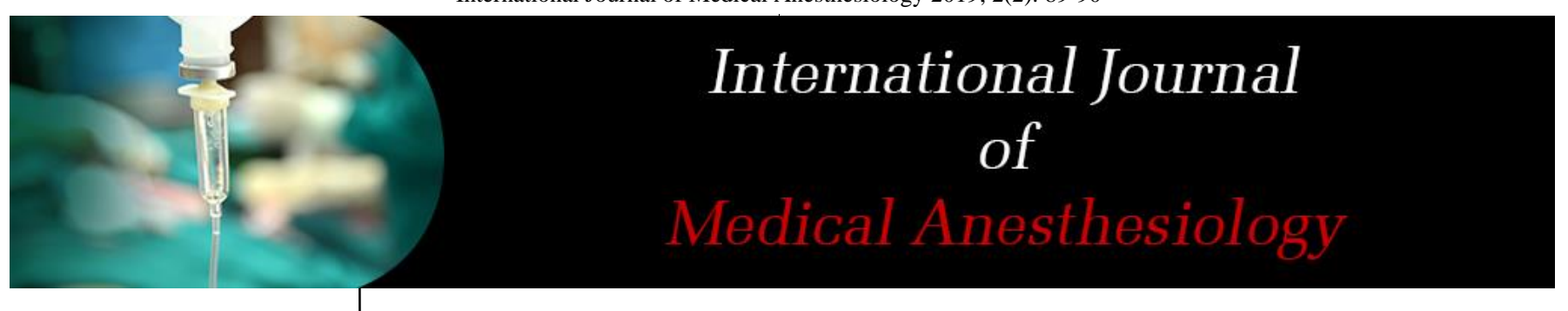

E-ISSN: 2664-3774

P-ISSN: 2664-3766

www.anesthesiologypaper.com

MA 2019; 2(2): 89-90

Received: 11-05-2019

Accepted: 15-06-2019

Dr. Pooja Agrawal

Resident, Anaesthesia

Department, Mahatma Gandhi

Mission Medical College,

Kamothe, Navi Mumbai,

Maharashtra, India

Dr. Vishwas Sathe

Professor, Anaesthesia

Department, Mahatma Gandhi

Mission Medical College,

Kamothe, Navi Mumbai,

Maharashtra, India

\section{Anaesthetic management of ASD with VSD with small right ventricle coming for non-cardiac surgery: A case report}

\author{
Dr. Pooja Agrawal and Dr. Vishwas Sathe
}

DOI: $\underline{\text { https://doi.org/10.33545/26643766.2019.v2.i2b.35 }}$

\begin{abstract}
Congenital birth defects are still placed first among common birth defects. Anaesthetist has to face great challenge in handling child with congenital heart disease coming for non-cardiac surgery. Here we present a case report of a 4year old male child with large ASD with VSD with TRICUSPID ATRESIA with pulmonary stenosis with small right ventricle who developed brain abscess due to bypass of venous blood from pulmonary circulation thus preventing the phagocytosis of the infective organism by the pulmonary macrophages. Anaesthetic goals in these patients is to maintain or increase the SVR, minimize PVR and provide mild cardiac depression.
\end{abstract}

Keywords: Brain abscess, Ventricular septal defect (VSD), Atrial septal defect (ASD), Tricuspid atresia, pulmonary stenosis, small RV, anaesthetic management

\section{Introduction}

Congenital heart disease are still placed first among common birth defects, occurs approximately 1 in 125 live births ${ }^{[1]}$. There is great challenge for anaesthesiologist in handling patient with CHD coming for non-cardiac surgery ${ }^{[1,2]}$. Factors important for anaesthetist are patients age, complexity of heart lesion, coupled with patients capacity to compensate, urgency of surgery and multiple co existing disease.

\section{Material and Methods}

A 4 year old male child presented with fever and convulsion which was examined and investigated as hydrocephalus? Brain abscess in our hospital. History was obtained from father, child was born at full term by normal delivery with birth weight of $2 \mathrm{kgs}$. Child had cried after birth. During antenatal Check up USG was suggestive of? CHD, mother was followed up with iron and folic acid tablets. On birth child had CTEV and CHD which was diagnosed on examination, on $2 \mathrm{~d}$ echo it was reported as ASD, VSD, tricuspid atresia, with pulmonary stenosis, with small right ventricle. Child had no distressing symptoms of CHD and so was not planned for surgery at birth. At age of 1 year during regular follow up for CHD and CTEV, child was diagnosed for oesophageal duplication cyst for which thoracotomy with excision of cyst was done. There was no developmental delay, he was immunised for age and was not on any medication.

At the age of 4 year child e was been planned for bilateral Glenn shunt surgery before which he developed fever with convulsions which suggested of hydrocephalus? Brain abscess for which he was brought to are hospital.

On examination the child was $4 \mathrm{~kg}$. He had clubbing, peripheral cyanosis. Heart rate is 150 , Respiratory Rate is $37 / \mathrm{min}$, BP was $90 / 40 \mathrm{~mm}$ of hg with SPO2 of $78-80 \%$ on room air. On examination of cardio vascular system, he had a pan-systolic murmur. Respiratory and Per abdomen examination was normal. On admission GCS was E4V1M1, with deviation of angle of mouth to right side, tone and reflexes - normal, power $4 / 5$ of the right side on investigation, Hemaoglobin was 14.8, Total leucocyte count was 28790, PT/INR 23/1.3, $\mathrm{Na} / \mathrm{k} / \mathrm{CL} 151 / 3 / 116$, CSF -wnl, blood group-A positive. Rest all investigation were within normal limit. 2decho showed situssolitus, large CV VSD left to right shunt, large OS ASD right to left shunt. Severeinfundibular and valvar PS gradient $67 \mathrm{mmhg}$. Normal LV function. Tricuspid atresia, RV smallish. ABG showed ph 7.36, pco2 38, po2 57, hco3 21 . A cardiologist opinion was sought and it was decided to do the surgery under high risk consent. On aadmissionchildspt/inr was $27.1 / 2.31$ with $\mathrm{Na} / \mathrm{k} 129 / 7.6$. It was corrected in picu with fresh frozen plasma at $10 \mathrm{ml} / \mathrm{kg}$ over $30 \mathrm{mins}$, injVit $\mathrm{k} 5 \mathrm{mg}$ iv once a day, with $3 \% \mathrm{nacl}$
Corresponding Auth
Dr. Vishwas Sathe

Professor, Anaesthesia

Department, Mahatma Gandhi

Mission Medical College,

Kamothe, Navi Mumbai,

Maharashtra, India 
@ $5.5 \mathrm{ml} / \mathrm{hr}$ over 24 hour and with $1 / 2 \mathrm{dns} 650 \mathrm{ml}+13 \mathrm{mlkcl}+\mathrm{inj}$ nahco320ml@55ml/hr. Child was electively intubated in picu on arrival in view of low gcs with ET $4.5 \mathrm{~mm}$. On discussion with neurosurgeon, craniotomy with abscess drainage was scheduled for emercency. The child was kept nil per oral for6hrs duration. Infective endocarditis prophylaxis was given to the child $1 \mathrm{hr}$ before the procedure. Child was shifted to ot on ambu bag with iv crystalloids started at $50 \mathrm{ml} / \mathrm{hr}$. All monitors including pulseoximetry, ecg, capnogram were attached. Baseline readings were recorded. Child was premedicated with Inj midazolam $0.4 \mathrm{mgand}$ inj fentanyl $18 \mathrm{mg}$ in titrated doses. Arterial line was secured in the left dorsalispedis artery and IBP was recorded throughout the surgery. Child was induced with injetomidate $2.7 \mathrm{mg}$ and injatracurium $9 \mathrm{mg}$. Anaesthesia was maintained with $02+$ air+sevoflurane. His saturation improved and maintained to $92-94 \%$ throughout the surgery. Surgery lasted for two hours with $30-40 \mathrm{ml}$ of blood loss, $150 \mathrm{ml}$ of urine output with total input of $250 \mathrm{ml}$ of ringer lactate. Child was shifted to picu sedated and paralysed. Post of period was uneventful and was extubated two days later and was followed up.

\section{Discussion}

Brain abscess is defined as an intra-parenchymal collection

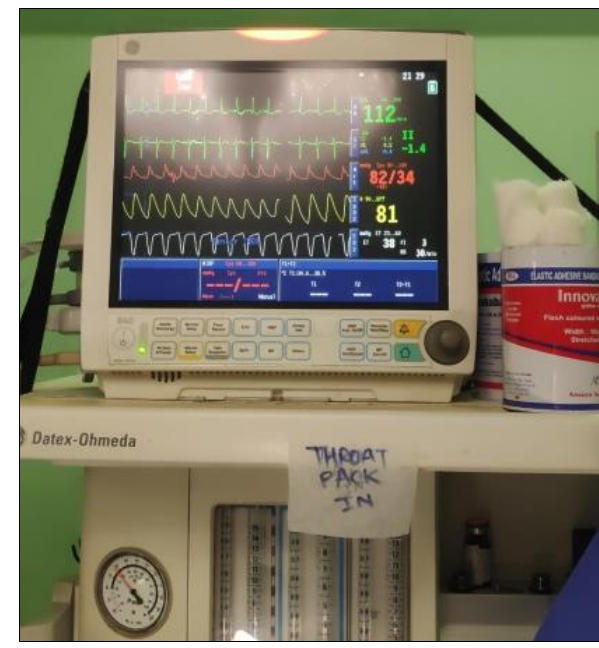

Fig 1: Intraoperative vitals

\section{References}

1. Anaesthetic Management of Tetralogy of Fallot Coming for Non-Cardiac Surgery: a Case Report Dilesh K1, K. Cheran 2, G. Gopi Kumar 3, Ittyerah P. Chandy. 2015; 4(23):4029-4032.

2. White MC. Approach to managing children with heart disease for noncardiac surgery. Paediatr Anaesth. 2011; 21:522-9. [PubMed].

3. An intra-cerebral abscess in a patient with Eisenmenger syndrome: An unusual case Casepanel Allan Hall Mark A.J. White Pasquale Gallo. 2016; 20:138-141.

4. Miranda HA, Leones SMC, Elzain MALF. MoscoteSalazar Brain abscess: current management J Neurosci. Rural Pract. 2003; 4(1).

5. Takeshita M, Kagawa M, Yato S, Izawa M, Onda H, Takakura A et al. Current treatment of brain abscess in patients with congenital cyanotic heart disease Neurosurgery. 1997; 41:1270-1279. of pus that occurs due to direct inoculation of septic foci into the brain or by the haematogenic or contiguous spread of infection [3, 4]. Brain abscess that presents with neurological deficitand raised ICP is an emergency, management includes the treatment of the abscess followed by the followed by the source of present recurrences. Our patient presented with congenital heart disease with ASO which was from right to left shunt, VSD from left and right shunt with pulmonary stenosis, leading to venous blood to bypass the pulmonary circulation, thus preventing the phagocytosis of the infective organism by the pulmonary macrophages $[3,5]$. There are prone to polycythemia that increases blood viscosity resulting in hypo perfusion of abscess in the brain and metabolic acidosis leading to seedling of the infective foci into the brain. It is a great challenge for anaesthetist as all commonly used induction agents have effect on SVR, PVR. In CHR, we need to maintain balance between SVR and PVR hence inhalational drugs dose and rate of drugs should be titrated.

\section{Result}

A 4year old child with large ASD, VSD with tricuspid atresia with pulmonary stenosis who developed brain abscess had an uneventful surgery and was successfully managed intra operatively under general anaesthesia.

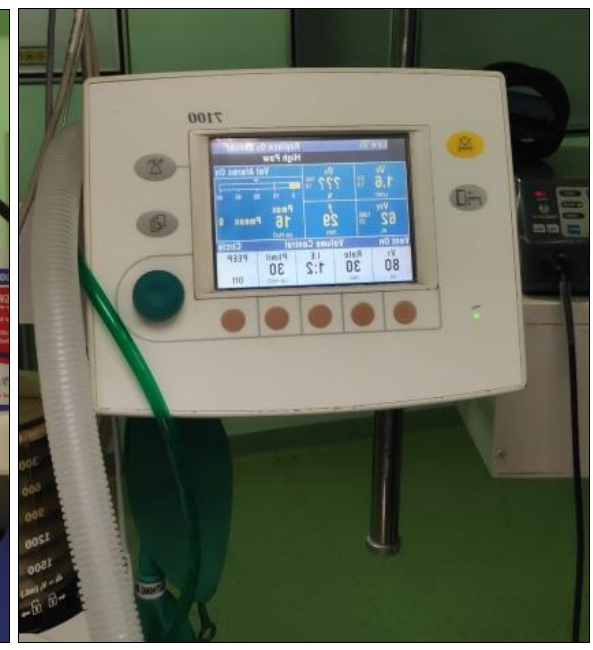

Fig 2: Ventilator setings for a $4 y r$ old child 